\title{
Estudiar la migración indígena. Itinerarios de vida de trabajadores agrícolas en el noroeste mexicano
}

\section{Studying indigenous migration. Life itineraries of farm workers in northwest Mexico}

Laura Velasco-Ortiz*

\begin{abstract}
The present work is a methodological exercise that tries to analyze the multiple logics that define the mobility and migration of indigenous people whose lives are connected with the development of agricultural labor exportation markets and the creation of new regions in the northern border of Mexico. The analysis is based on biographical interviews considering mobility, which allows us to observe the multiple logics that organize the movement of laborers as well as migration that takes us to observe the settlement process in new places.
\end{abstract}

Keywords: migration, mobility, agricultural work, life pathways.

\section{Resumen}

La presente investigación es un ejercicio metodológico en el cual se analizan las múltiples lógicas que definen la movilidad y la migración de poblaciones indígenas, cuyas vidas se interrelacionan con el desarrollo de los mercados de trabajo agrícola de exportación y la creación de nuevas regiones en la frontera norte de México. El análisis de dicha movilidad parte de entrevistas biográficas, lo cual nos permite observar a partir de qué condiciones se suscita el desplazamiento de los jornaleros y la migración, así como el proceso de asentamiento en nuevos lugares.

Palabras clave: migración indígena, movilidad geográfica, trabajo agrícola, itinerarios de vida. 


\section{Introducción}

En las últimas tres décadas la migración indígena ${ }^{1}$ parece haber cobrado una mayor importancia para los estudiosos de la migración interna e internacional en México. ${ }^{2}$ La diversidad disciplinaria de las investigaciones ha enriquecido el campo de los estudios indígenas con nuevos temas y retos metodológicos, especialmente en lo que se refiere a las amplias corrientes migratorias de indígenas que tienen lugar en el interior del país y Estados Unidos. Los estudios realizados sobre el tema han documentado un comportamiento migratorio específico de las poblaciones indígenas. Se ha establecido una asociación entre la movilidad geográfica de estas poblaciones con ciertos mercados de trabajo, particularmente con el de la agricultura de exportación y el comercio informal en las ciudades; así como, incipientemente, con la industria maquiladora (Velasco, 2005; Velasco, 2010; Lara-Flores, 2003).

Esta investigación presenta un ejercicio metodológico que analiza la movilidad y migración de poblaciones indígenas, cuyas vidas se interrelacionan con el desarrollo de los mercados de trabajo agrícola de exportación y la creación de nuevos núcleos de asentamiento en la frontera norte de México.

El esfuerzo metodológico que subyace a este artículo se relaciona con el supuesto planteado por Lara-Flores (2008) sobre el nexo entre la movilidad de los trabajadores y la flexibilidad extrema en los contratos laborales, es decir, la contratación y los despidos arbitrarios con los que operan los mercados de trabajo, lo cual provoca a los trabajadores una alta condición de vulnerabilidad.

Ante la falta de estructuras institucionales de asistencia y protección ¿cuáles son los mecanismos con los que los trabajadores resuelven esta situación cotidianamente?, ¿cómo subsisten día a día en condiciones de movilidad precaria y explotación laboral? Este artículo plantea algunas respuestas a través del estudio de sus relatos de vida, poniendo especial atención en sus itinerarios.

\section{Aclaraciones conceptuales y metodológicas}

El concepto de movilidad geográfica da la posibilidad de pensar en una amplia variedad de traslados humanos que puede incluir a la migración

\footnotetext{
${ }^{1}$ La definición de la población indígena dentro de esa corriente migratoria se basa en el criterio de lengua indígena: pertenencia a un hogar con algún hablante, o bien, nacido en un municipio de estados como Oaxaca o Guerrero cuya población sea predominantemente indígena.

${ }^{2}$ Ver Rubio et al. (2000) y Fox y Rivera (2004).
} 
como un fenómeno específico. En un esfuerzo por dialogar con ambos conceptos es útil su distinción.

La movilidad geográfica se define como todo desplazamiento humano independientemente de los motivos, condiciones y consecuencias; en ella se pueden incluir el turista, el ejecutivo, el jornalero o el refugiado. En tanto, la migración tiene que ver con cualquier desplazamiento con expectativas de cambio en el plano individual o familiar -puede ser laboral o no- e impacta las condiciones de residencia y empleo en primera instancia y de integración social en segundo término.

Así, la movilidad geográfica incluye distintos tipos de desplazamientos humanos (Heyman, 2012), ${ }^{3}$ entre ellos las migraciones temporales nacionales e internacionales. En los distintos flujos de la amplia movilidad humana contemporánea confluyen los commuters laborales o estudiantiles, los compradores, los visitantes de corta estancia y los migrantes laborales y familiares de ida y de retorno (deportados y no).

La distinción entre la movilidad humana y la migración es ciertamente compleja debido a que puede haber mezcla de patrones en la historia de una sola persona como bien lo ilustra Heyman, tal es el caso de estudiantes commuters en la frontera que combinan sus estudios con el empleo en el extranjero, así que, ‘hablamos de movilidad estudiantil o de migración laboral? Esta dificultad lleva a Heyman (2012) a considerar que el concepto de migración parece un asunto de clase y edad.

Sin embargo, dado la enorme tradición en los estudios de migración en América Latina y Norteamérica es difícil presindir de los conceptos y categorías producidos por ese cuerpo de conocimiento, específicamente de aquellos relacionados con la búsqueda de estabilidad, como es el de asentamiento residencial que permite considerar el fenómeno de permanencia en los lugares de destino de un número cada vez mayor de trabajadores temporales de origen indígena y de otros estratos sociales.

Un balance entre la movilidad y el asentamiento ha sido señalado por Cresswell $(2012)^{4}$ como una necesidad analítica reciente que urge a reconocer los estados de espera o sedentarismo sin desconexión con la movilidad.

En este artículo combinamos ambas perspectivas analíticas: la de la movilidad, para observar las múltiples lógicas que organizan el desplazamiento de los jornaleros agrícolas y, la de migración, para observar la dinámica de asentamiento de dichos trabajadores en nuevos lugares. La movilidad y el asentamiento son procesos de la dinámica de reproducción

\footnotetext{
${ }^{3}$ Heyman (2012: 427) define a la movilidad como: "cualquier tipo de patrón de movimiento humano o no (bienes o información), en la que lo importante es su relevancia social y cultural...”.

${ }^{4}$ Cresswell, 2013 y 2014.
} 
de poblaciones que viven en desplazamiento continuo y a la vez creando nuevos núcleos de asentamiento.

A partir de la perspectiva biográfica se analizan las tres dimensiones que surgen recurrentemente en la caracterización de la movilidad y migración indígena al noroeste mexicano y el suroeste estadounidense: su temporalidad, espacialidad y especialización laboral.

La literatura sobre el tema ${ }^{5}$ ha documentado desde los años sesenta del siglo xx la existencia de una migración indígena a las regiones agrícolas del noroeste mexicano. Según Lara-Flores (2008), los principales estados de recepción de los trabajadores agrícolas en el noroeste mexicano son Sinaloa (35.8\%), Baja California (32.7\%), Sonora (6.6\%) y Baja California Sur (6.2\%). ${ }^{6}$ Estos inmigrantes tienen una movilidad y residencia estacional en circuitos geográficos más o menos bien definidos por el mercado agrícola y una especialización laboral como jornaleros en cultivos específicos. Así, este migrante es el jornalero que vive atado a la lógica de producción del mercado agrícola.

Un reto de investigación y metodológico es analizar la forma en la que dichos jornaleros contienden entre las opciones laborales que les ofrecen esos mercados a lo largo de sus vidas. Con el estudio biográfico se persigue un acercamiento a las opciones de vida que experimenta el individuo en su trayecto vital y la forma de enfrentar el funcionamiento de los mercados transnacionales. La lógica con la que los individuos se mueven en ese mercado representa una perspectiva indispensable para poder comprender mejor el fenómeno migratorio y sus cambios.

El material de apoyo para este análisis preeliminar está constituido por una base de datos de 100 entrevistas realizadas entre 2003 y 2007 , bajo la perspectiva biográfica en torno a tres ejes: movilidad geográfica, trabajo y proceso de asentamiento en el valle de San Quintín. ${ }^{7}$ Para este estudio se tomaron sólo 10 casos y entre las características de los entrevistados destacan que la media de edad es de 40 años, la mitad son hombres y la otra mitad, mujeres; nueve son bilingües (español y lengua indígena): cinco mixtecos, tres triquis y un zapoteco; sólo uno habla español. Todos ellos llegaron al valle de San Quintín entre 1970 y 2000 , la mitad arribó a esta región en la década de los ochenta. En los años que

${ }^{5}$ Arroyo-Sepúlveda, 2003; Barrón, 1994; Lara-Flores, 2003; Lara-Flores y Carton, 2003 y Zabin, 1993.

${ }^{6}$ Carton y Lara-Flores (2004) realizaron una encuesta a 8,117 hogares de jornaleros agrícolas migrantes en campamentos y cuarterías de las regiones agrícolas más desarrolladas en los estados de Sinaloa, Sonora, Jalisco y Baja California Sur, entre 1998 y 2000.

${ }^{7}$ Las entrevistas se realizaron en el marco del proyecto de investigación "Migración, trabajo agrícola y etnicidad: la relación entre lo local, nacional y global en el valle de San Quintín (20032006)" con apoyo de Conacyt, donde participan -además de la autora Marie Laure Coubès- Christian Zlolniski, Abbdel Camargo, Susana Vargas y Juan Manuel Ávalos. 
se realizaron las entrevistas el grupo de estudio eran jornaleros y jornaleras y vivían en alguna de las colonias de las delegaciones de Vicente Guerrero y San Quintín. ${ }^{8}$

Como se observará más adelante, estas entrevistas tuvieron como fin estudiar cada una de las dimensiones de la migración: la temporalidad, la espacialidad y la especialización laboral. El objetivo es dar un primer paso en la asociación de dichas dimensiones a episodios vitales, ya sea individuales, familiares y comunitarios, esto con el propósito de comprender los factores que definen el comportamiento de los migrantes, a partir de esos tres parámetros.

En el análisis prevalece el interés metodológico por encontrar algunos patrones o comportamientos típicos que permitieran caracterizar la complejidad de la migración indígena y que, a su vez, fueron la base para la redacción de un cuestionario que se aplicó posteriormente en forma de encuesta (Ebimre, 2005) y del cual se utilizaron algunos datos obtenidos. ${ }^{9}$

Para la exposición de los resultados utilizo el caso de Julián, ${ }^{10}$ un hombre originario de la región mixteca de Oaxaca, hablante del mixteco y español y dedicado al trabajo agrícola en la mayor parte de su semblanza laboral, con una trayectoria de movilidad de cerca de 40 ańos, la cual incluye las rutas que caracterizan a las corrientes migratorias oaxaqueñas: migración al sur en los ańos cuarenta y luego al noroeste, asentamiento en San Quintín y migración internacional en circuito temporal. En su historia migratoria encontramos las diferentes lógicas presentes en los demás casos, permitiendo ilustrarlas en un continuo biográfico (Julián, 2004).

\section{Migración, movilidad, asentamiento y especialización laboral de los indígenas en el valle de San Quintín}

En el 2000 la población del valle de San Quintín ascendía a 74,727. De acuerdo con la Ebimre (2005), 61.3\% de la población había nacido en Oaxaca y $34.6 \%$ en el estado de Guerrero, y en porcentajes menores en Sinaloa (4.2\%) y en Baja California (6.3\%). Además de un origen regional hay un origen étnico, ya que $20 \%$ de los habitantes en ese mismo año hablaban alguna lengua indígena, la mayoría el mixteco y en menor grado el triqui y zapoteco.

${ }^{8}$ Estas dos delegaciones concentran $70 \%$ de la población de todo el valle. La definición del valle de San Quintín no obedece a una demarcación política administrativa, pues abarca cuatro delegaciones: Punta Colonet, Camalú, Vicente Guerrero y San Quintín.

${ }^{9}$ Las primeras entrevistas sirvieron para la elaboración de una encuesta representativa de corte biográfico sobre movilidad, residencia y empleo en el Valle de San Quintín. Dicha Encuesta Biográfica de Movilidad Residencial y de Empleo en San Quintín (Ebimre) se realizó entre mayo y junio de 2005; sobre el diseńo de ésta ver Coubès (2005).

${ }^{10}$ Los nombres que utilizo son seudónimos. 
El arribo de los migrantes a esta región puede comprenderse a partir de la historia de la migración indígena oaxaqueña hacia el noroeste de México en los años sesenta en un contexto histórico que planteo en otra investigación a partir de seis etapas (Velasco, 2002). El inicio de la migración oaxaqueña moderna ${ }^{11}$ se puede establecer en los años cuarenta con las migraciones agrícolas para el corte de caña en Veracruz y la incipiente incursión de los pobladores de la región mixteca en el Programa de Braceros que estableció México con Estados Unidos.

En la década de los cincuenta las rutas migratorias incorporan a las ciudades de Oaxaca, Puebla y Distrito Federal como destinos urbanos, y para los sesenta se establece la conexión con la migración urbana a las ciudades y con otras zonas agrícolas como las de Veracruz y Morelos. En la misma década la ruta al noroeste reúne los flujos de los pueblos originarios y de puntos intermedios, ofreciendo alternativas de trabajo temporal a una gran cantidad de pobladores de distintas localidades indígenas en la región y en otros lugares donde habían emigrado.

El destino particular a la región del valle de San Quintín se ubica en esta ruta noroeste que desde su inicio se conecta con la migración internacional al estado de California en Estados Unidos. Esta nueva ruta de la agricultura moderna del noroeste mexicano se asocia con un mercado de trabajo temporal en dicha actividad. Sin embargo, en los años ochenta es posible observar un proceso de asentamiento en colonias periféricas a las ciudades de la frontera noroeste mexicana, así como en colonias aledańas a los campos agrícolas de la misma región.

En la década de los setenta, el valle de San Quintín siguió los pasos de otras regiones agrícolas de Sonora y Sinaloa al utilizar el reclutamiento a distancia de trabajadores temporales para la incipiente agricultura de exportación. Durante la década de los ochenta y principios de los noventa los trabajadores, en su mayoría migrantes, se hospedaban en viviendas colectivas, en campamentos construidos por los rancheros o empresarios dentro de su propiedad, o bien, rentaban pequeños cuartos en los poblados vecinos.

Esa última década (los noventa) parece condensar una serie de cambios que confluyen en la transformación del valle. A mediados de la primera década del siglo xxi era cada vez más común encontrar campamentos agrícolas vacíos o en proceso de desinstalación, lo que podría llevar a un observador inexperto a concluir que hay una debacle en la economía agrícola de la región.

\footnotetext{
${ }^{11}$ Enfatizo la migración moderna para distinguirla de la movilidad regional de los pobladores de la región mixteca, previas a la etapa de industrialización y modernización en México.
} 
No obstante, un recorrido por las nuevas colonias populares de este valle durante horas estratégicas como las cinco o seis de la mañana obliga a relativizar tal conclusión, ${ }^{12}$ pues a esas horas se puede observar una ebullición en la vida de las colonias: los trabajadores se apresuran a abordar los camiones escolares llevando baldes blancos para la pizca y las bolsas con su comida preparada. Son los nuevos jornaleros agrícolas que ya no se hospedan en los campamentos o cuarterías, sino en las colonias populares donde poseen un lote en proceso de regularización con posibilidad de acceder a los servicios de luz y agua.

Lo anterior es una señal de los cambios sucedidos en las dos últimas décadas en el valle; para los términos de este artículo sólo enfatizaré dos principales cambios que, aunque parezca que corren en forma paralela, se relacionan estrechamente: el primero de ellos se da en el terreno de la tecnología de la producción y el segundo en la organización del trabajo.

Desde su origen, la producción agrícola regional utilizó la tecnología de riego; sin embargo, en la década de los noventa sucede un cambio sustancial con la aparición del riego y la fertilización por goteo, la doble línea en surco y el invernadero; este último se extendió a través de la plastificación del surco a cielo abierto y el invernadero a cielo cerrado en el valle. ${ }^{13}$ Según algunos de los empresarios estas nuevas tecnologías, aunque al principio fueron vistas con recelo, permitieron enfrentar de mejor manera el problema de la escasez de agua en la región.

En la producción, los cambios tecnológicos permitieron ampliar los periodos a un año completo al introducir los cultivos de invierno, así como a incrementar el rendimiento por hectárea como resultado del mejoramiento de la semilla y de la mayor concentración de plantas por espacio. Como lo señala el señor José, encargado de uno de los ranchos más grandes en el valle, en los años ochenta ya había un incremento de la producción por planta y por surco, el cual también implicó cambios en la organización de la cosecha y el empaque: se sustituyeron las cajas de madera por tinas de plástico directamente a los surcos y se empezó a cosechar el tomate de acuerdo con su color.

denominamos colores: uno era verde, dos poquito pintado, tres ya como $1 / 4$ del fruto con color, cuatro medio y medio cinco; pues ya el tomate color de rosa y seis un color que ya está en estado bueno para empacar estaba muy maduro; empezamos a determinar todos esos detalles y em-

\footnotetext{
${ }^{12}$ A principios de 2004, un recorrido por el valle nos permitió documentar 62 colonias de residentes en las delegaciones más pobladas de la región: Vicente Guerrero y San Quintín.

${ }^{13}$ Entrevista a Juan Manuel Llamadas (2000), delegado del Programa Nacional de Jornaleros Agrícolas en el valle de San Quintín, realizada por Laura Velasco, Marie Laure Coubès y Christian Zlolniski. Esto también lo confirmó el profesor Andrés Cruz, director de la escuela Revolución Mexicana, Nuevo San Juan Copala, en entrevista realizada por Laura Velasco (2004).
} 
pezamos a controlar [la cosecha]; por decir algo, en la mańana al jefe de operaciones de campo: se le decía "haz una estimación de lo que vamos a cosechar hoy...” (José, 2005).

Los cambios tecnológicos en la siembra y la cosecha impactaron en la temporalidad, diversidad e intensidad de la producción a lo largo del año. Los datos de siembra y producción a fines de la década de los noventa ya registran claramente las temporadas de producción del año completo.

Entre 1998 y 1999, así como en 2001 y 2002 la superficie sembrada en el ciclo primavera-verano se redujo en un dramático 40\% (de 10,000 a 6,000 hectáreas). En tanto la producción en ese mismo periodo se redujo en 35\% (351,000 a 230,000 toneladas). Esa reducción también se registró en el ciclo otońo-invierno, pero tuvo una caída menos drástica y ofreció una oportunidad de empleo a un segmento de los trabajadores desocupados por el fin del ciclo primavera-verano. ${ }^{14}$

El rendimiento por hectárea durante la misma década se mantuvo constante e incluso registró un incremento constante los dos ciclos. A la vez estos cambios tecnológicos permitieron lidiar con la contracción de la superficie y de la producción agrícola de fines de los años noventa, ya que facilitaron la ampliación de los periodos de producción de algunos cultivos y la producción de una mayor variedad de cultivos en invierno.

Del lado de los trabajadores, tales cambios suscitaron una notable disminución de la población en los campamentos e incentivaron el asentamiento de muchos de esos migrantes estacionales en las colonias recién inauguradas en el valle, con posibilidades de trabajar todo el año.

El proceso de asentamiento es el segundo cambio que me interesa enfatizar aquí, en un primer acercamiento, éste se expresó en la toma o compra de terrenos en forma colectiva por grupos de trabajadores que lentamente se habían instalado en los campamentos temporada tras temporada, o bien, vivían en las cuarterías de renta en los poblados. Este asentamiento generó, a su vez, una serie de transformaciones de índole social y cultural.

La vida familiar y comunitaria pudo recrearse en otras condiciones al separar los espacios de producción y los de reproducción de la fuerza de trabajo, a la vez que abrió la posibilidad de otras ocupaciones. En 1989, $66.7 \%$ de la población de la región vivía en campamentos, mientras que diez años después, en 1999, sólo era 33\% de la población total (Velasco, 2005: 71).

Dicha transformación puede observarse en el perfil de ocupación de la población residente en las colonias del valle de San Quintín. Entre 1990

\footnotetext{
${ }^{14}$ Datos procesados por el proyecto Colef-Conacyt, "Migración, trabajo agrícola y etnicidad" con base en Sagarpa. Programa Agrícola (1992-1993, 1996-1997 a 2003-2004).
} 
y 2000 se registra un cambio en la proporción de la población ocupada en el sector primario de 61\% a 54.6\% (INEGI, 1990-2000). El sector primario se refiere básicamente al empleo en la agricultura, el secundario en la industria, particularmente la textil, y el terciario en el comercio y los servicios que, después de la agricultura, ocupan a la proporción más alta de los sanquintenses (INEGI, 1990 y 2000).

Estos últimos datos reflejan el incremento de la presencia gubernamental a nivel municipal y estatal en los años noventa, así como el incremento de un segmento de la población de nivel profesional ocupando puestos en la burocracia institucional local; el florecimiento en las colonias de las tienditas de alimentos, comercios de ropa, artículos electrodomésticos y productos para la construcción; sin embargo, esa incipiente diversificación de la actividad económica en el valle no ha desplazado a la agricultura industrial como la base de la acumulación económica en la región, por lo que la dinámica de los otros sectores funciona en torno a la vitalidad de esa actividad.

Por ejemplo, en las pequeñas tienditas de las colonias las personas pagan con los cheques expedidos por las compañías agrícolas y los propietarios de esas tienditas a la vez pagan sus deudas a los proveedores con los mismos cheques. De tal forma que cuando hay problemas de liquidez de los cheques, sucede un efecto encadenado que involucra a los empresarios agrícolas, a los pequeños comerciantes y a las empresas transnacionales Sabritas o Coca Cola. ${ }^{15}$

La tendencia a la diversificación ocupacional parece diferenciar a la población del valle, ya que los nativos se ocupan menos en las labores agrícolas, mientras que son los migrantes, sean de estratos étnicos o no, los que realizan estas actividades, es decir, los nacidos en el estado de Baja California se dedican en mayor proporción a otras actividades distintas de la agricultura respecto de otras categorías sociales, como son los migrantes y los indígenas.

De tal manera que hemos llegado a la conexión entre la migración, el trabajo agrícola y la condición de ser indígena. Estos datos confirman la hipótesis de la especialización laboral de la población indígena en el trabajo agrícola y en la posición de peón, relación que se ahonda más en los campamentos. ${ }^{16}$ En 2003, 96.2\% de la población hablante de lengua indígena que vivía en campamentos se dedicaba a la agricultura; mientras que un año antes en las colonias esa proporción era de $83.9 \%$, pues sur-

${ }^{15}$ No es extraño que en las movilizaciones por la suspensión de salarios en los años noventa, los trabajadores no sólo quemaron los empaques sino también saquearon las tiendas donde no les fiaban alimentos por la falta de cheques.

${ }^{16} \mathrm{Habla}$ una lengua indígena $34 \%$ de la población mayor de cinco ańos que vive en campamentos, en tanto en las colonias ese porcentaje es de 16\%. Encuesta Sociodemográfica y Migración del valle de San Quintín (Conepo-Colef, 2002-2003). 
gieron para este último grupo ocupaciones como comerciantes y trabajadores ambulantes, en la industria y en otros servicios, en orden de importancia.

Así, el proceso de asentamiento aún no ha logrado modificar sustancialmente la especialización laboral de los indígenas migrantes en la actividad agrícola. No obstante, dado lo nuevo en su proceso de asentamiento, el hecho de que cerca de $20 \%$ de la población económicamente activa tenga una ocupación distinta a la agricultura es un hallazgo importante de observar en su evolución.

A continuación, se tratan de explorar los cambios que ha traído el asentamiento a la dinámica del empleo agrícola en la región. Las transformaciones en la producción no han alterado la característica básica de la industria agrícola cuyo tiempo de producción es distinto al tiempo del trabajo, ${ }^{17}$ es decir, éste no se realiza de forma constante en todo el ciclo de producción, sino que se concentra en ciertos momentos en forma intensiva especialmente en los tiempos de siembra y cosecha; esto implica que se requiere fuerza de trabajo en cuotas diferenciales a lo largo del ciclo de producción y, por lo tanto, flujos de trabajadores por temporadas.

Sin embargo, esos cambios en la producción, particularmente los tecnológicos, sí han traído transformaciones en la organización del trabajo, tales como: a) la diversidad de cultivos; b) los diferentes tipos de contrato (por jornada, tarea o pieza; c) la heterogeneidad de patrones y, d) el cambio del papel de los intermediarios laborales al decaer el enganche a distancia y surgir el reclutamiento en colonias.

Para ilustrar estos cambios es útil el caso del señor Moisés, un trabajador del campo que tiene una relación laboral de muchos años con un patrón, la empresa en la cual labora se dedica principalmente al cultivo de la fresa, de tal manera que en la temporada que va de otońo a invierno se intensifica el trabajo para él y su familia (esposa e hijos).

En abril decae la producción de fresa e inicia el periodo de otros cultivos como el tomate, el cual se extiende durante todo el año en tres etapas, como menciona el señor Moisés: "en su primera etapa, los meses de mayo a julio; segunda etapa de agosto a octubre y la tercera etapa de noviembre a diciembre. Durante esos meses pueden contratarse con otros patrones en el corte de tomate y a la vez seguir trabajando por día en contratos de tarea para la preparación de los terrenos para el cultivo de la fresa”.

Otro caso ilustrativo es el de Rosa, una trabajadora agrícola asentada en la colonia San Juan Copala, quien narra que en noviembre de 2004 los camiones que arribaban a las colonias llevaban a los trabajadores a

\footnotetext{
${ }^{17}$ Martínez-Veiga (2001: 45-47), en su estudio del ejido, considera que este desajuste entre el tiempo de producción y de trabajo trae consecuencias particulares a la organización de la labor agrícola.
} 
distintos ranchos: "usted puede elegir a cuál subir si no tiene contrato por temporada... puede irse a la tarea de la fresa... por ejemplo, a levantar plástico o poner estaca... o bien, ir a cortar tomate... pero el tomate es muy pesado porque los botes son grandes... prefiero ir a levantar plástico, donde pagan por tarea...”. Es decir, la oferta de trabajadores se organiza por colonia, ya no por campamento, y esto da flexibilidad a los empleadores y a los empleados, una vez roto el vínculo residencia-empleo que existía en los campamentos.

El cambio del perfil de los intermediarios laborales es también una de las modificaciones en cuanto a la organización del trabajo se refiere, pues ha surgido en el valle una compleja variedad de contratistas locales que organizan la fuerza de trabajo, desempeñándose como choferes y, en ocasiones, como mayordomos en los campos de cultivo. Esta novedad en la organización del trabajo ha debilitado la contratación a distancia y, por lo tanto, la movilidad espacial de los trabajadores.

En los últimos treinta años ha cambiado el traslado de los trabajadores: en los años setenta era usual transportarlos en grupos, en líneas de camión o camiones de los patrones (cuando era la misma región noroeste), y para el traslado del campamento al campo podía hacerse en camiones de redilas. Una vez que los trabajadores se asentaron se incrementó el uso de transportistas que, solos o en forma organizada, se encargan de abastecer a las empresas de trabajadores de las colonias.

Como lo menciona Rogelio, presidente del Consejo de Administración de Autotransportes del Valle:

\begin{abstract}
los transportistas son un gremio que trabaja en conexión con las empresas, con unidades vehiculares propias que comúnmente son camiones de escuelas de segunda mano traídos desde Estados Unidos. Muchos de los camioneros eran empleados de los ranchos y se independizaron para manejar su propia unidad y tener un contrato asociado a un número de trabajadores en cuotas adecuadas a las necesidades de las empresas.
\end{abstract}

El asentamiento no sólo ha afectado la organización del trabajo sino las condiciones de vida de los trabajadores. Los nuevos residentes del valle ya no regresan a sus lugares de origen o circulan en el noroeste, sino alternan entre empresas y cultivos para tener empleo durante todo el año, e incluso realizan también otras actividades en otros sectores o emigran hacia Estados Unidos para incrementar los ingresos que provienen de la agricultura en el valle.

Un dato importante según el presidente del Consejo de Administración de los Transportistas es que los salarios eran más bajos cuando traían a los trabajadores desde sus lugares de origen debido a que les cobraban el 
viaje. Además, los empresarios confiaban en que aceptarían dicho pago puesto que sentían cierta lealtad, Rogelio continua

Sale más barato. Es negocio traer gente de fuera. Yo traigo gente de fuera y le pago menos. Y como están contratados hay una obligación moral de la gente de quedarse con quien los trajo... De algunos. Vamos a hablar de la mitad; vamos a pensar que traen 50 y se van 25 , se quedan $25 \ldots$

Es negocio... Si yo vengo a un rancho que me contrata, me va pagar quizá a 2.50 la cubeta... Más bien, 2.25 la cubeta... de la pizca de tomate... 2.25. Pero si me voy a un rancho que no me contrató porque yo llegué solo, me va a pagar tres pesos la cubeta porque ese no le da... no le da cuarto, no le da escuela, no le da nada... pero le da tres pesos por cubeta; 50 centavos más. La gente hace 100 cubetas; 80,100 cubetas al día. 50 centavos, 100 cubetas por día. ¿Cuánto es a la semana? ¿Cuánto es en los cuatro o cinco meses? ¡Es un dineral! A final de cuentas es negocio para los ranchos grandes traer gente contratada porque les pagan menos. Y si invierten quinientos o seiscientos mil pesos trayendo gente... Entre trayéndolo y dándole servicios dejan de pagar tres y cuatro millones de pesos... Entonces es negocio traer gente; la verdad, es negocio...

Esto explica porqué los trabajadores están convencidos de asentarse, pues les trae mayores beneficios como tener una vivienda con servicios y escuela para los niños, así como ganar tal vez esos 50 centavos más. Así, el trabajador libre puede reapropiarse de su mano de obra y desplazarse desde su nueva residencia a distintas empresas, campos, actividades y cultivos a lo largo de un año. Considerando también la migración a Estados Unidos que toma como nuevo punto de partida la región de San Quintín, entonces a esa movilidad entre campos en el valle se suma la movilidad en campos de California, Oregón o Washington.

Vista desde la perspectiva del lugar de asentamiento, pareciera que está sucediendo un cambio fundamental en la vida de los indígenas migrantes que llegaron a San Quintín como trabajadores agrícolas y que formaron parte de las corrientes migratorias de los años ochenta. Una pregunta posible es ¿Cómo contienden los trabajadores con estas transformaciones?, ¿cómo sus lógicas de reproducción social y cultural se adaptan a estas nuevas condiciones de operación de un mercado de trabajo que integra mano de obra itinerante y asentada?

\section{Múltiples lógicas vitales: tiempo, espacio y relaciones sociales}

El estudio biográfico se utiliza como una vía para entender estos cambios estructurales desde la experiencia de vida de los indígenas que actualmente laboran en la agricultura de esta región. Uno de los supuestos más evidentes, con el análisis aquí presentado, es que existe un cambio en la manera cómo influye el tiempo y el espacio en el proceso de asentamien- 
to, ya que estos indígenas en su mayoría llegaron temporalmente al valle en los años noventa. ${ }^{18}$

Con el material biográfico cualitativo es posible decir que el tiempo de residencia en una colonia no es suficiente, sino que se acompaña con otros indicadores como el acceso a un terreno, la construcción de una casa y el pago de servicios urbanos. El asentamiento aparece como un proceso colectivo antes que individual, es decir, sucede cuando el grupo familiar se reencuentra en un lugar específico y se pasa del campamento a la colonia. Un aspecto importante de las migraciones indígenas es que lo colectivo no sólo incluye a la familia, sino a la comunidad local tanto étnica como de paisanaje.

Como lo señala Acebo (1996), el apego al nuevo territorio se da a nivel práctico y simbólico e implica una experiencia totalizadora, la cual se nutre de las lógicas individuales pero a la vez crea algo que va más allá de esa individualidad. Scudder (1985) considera que el éxito de un asentamiento se da cuando los colonos desarrollan un sentido de arraigo y pertenencia, yendo más allá de la satisfacción de necesidades; el arraigo no elimina las interacciones con los lugares de origen y con otros lugares de migración, donde se dispersan los familiares y miembros del pueblo o localidad.

El estudio biográfico nos permite comprender la complejidad del proceso de asentamiento y de movilidad y, al mismo tiempo, entender las estrategias de vida de estos individuos para resistir y adaptarse a la lógica de los mercados de trabajo y no sólo observarlos como trabajadores en constante movimiento, sino en un proceso de reconstitución como sujetos históricos.

$\mathrm{Al}$ analizar el conjunto de entrevistas biográficas se advierte que, a lo largo de su vida, el individuo experimenta la migración como un conjunto de movimientos geográficos con cambios de residencia. Esto fragmenta su experiencia de vida en lugares con relaciones sociales distintas y a las cuales él o ella les da sentido en su narración biográfica: a) la salida con algún familiar o paisano; b) el viaje acompañado con familiares o paisanos; c) los arribos múltiples a distintos lugares (que a veces incluyen ciudades) con cortas estancias, y d) la llegada al valle de San Quintín con algún familiar o paisano.

En este punto, las estrategias de encuentro son una constante y, después de un tiempo, se da un cambio de residencia que puede alternar con movilidades a otros lugares del noroeste o de Estados Unidos y con la autonomía residencial, ya sea a través de la renta o de posesión de un

\footnotetext{
${ }^{18}$ Del total de la población encuestada de las colonias en 2003, cerca de 70\% llegó en la década de los ochenta y noventa del siglo xx (Conepo-Colef, 2003).
} 
terreno en una colonia. En este proceso, los ejes temporales y espaciales siempre están mezclados con lo laboral y familiar, agregándose cada vez más la dinámica residencial en el nuevo lugar.

Uno de los retos de estudiar el proceso de asentamiento en estas poblaciones consiste en comprender la importancia que la movilidad geográfica ha tenido en la constitución de alternativas y horizontes de vida. A continuación hacemos un ejercicio con información cualitativa tomada de una entrevista realizada a un jornalero indígena que, por su larga historia, nos permite analizar la complejidad de sus procesos migratorios a lo largo de su trayectoria vital, tratando de distinguir las lógicas sociales de su movilidad.

\section{La multitemporalidad de la movilidad y migración}

La temporalidad de los movimientos migratorios se ha utilizado para distinguir tipos de migrantes, tales como los temporales, circulares o definitivos. En este apartado se expone ese supuesto con la idea de que las diferentes temporalidades nos permitan distinguir experiencias de vida constituidas por que llamaremos episodios migratorios, más que individuos. Los distintos movimientos geográficos pueden ser experimentados por el mismo individuo a lo largo de su biografía como migrante.

Este análisis nos permite diluciar que los entrevistados tienen una historia de movimientos geográficos con temporalidades distintas. En el conjunto de casos, las edades de los individuos van de los 31 a los 61 años, por lo que para evaluar mejor sus tiempos de movilidad, separé a los que tienen entre 31 y 40 años para estudiar una generación de migrantes: este grupo tiene en promedio una historia de movilidad de 22 años; es decir, la mayor parte de su vida fuera de su lugar de origen y con movilidades distintas.

De entre los entrevistados ninguno experimentó únicamente un desplazamiento geográfico a lo largo de su vida, incluso aquellos ya nacidos en el valle de San Quintín. Es difícil distinguir el tiempo del lugar en los movimientos migratorios, debido a que el lugar se vuelve una marca temporal del ciclo de vida. Un movimiento siempre implica un lugar de salida y uno de llegada, así como una duración. Los movimientos geográficos incluyen lugares diversos en un circuito específico en el territorio mexicano y extranjero.

A continuación se analizan esos movimientos en función de su temporalidad, tratando de rastrear la lógica social a la que obedecen. La referencia general de estas temporalidades fue la edad del individuo y, por lo tanto, su biografía total. 
Cada uno de estos tipos de movilidad parecen significar experiencias de vida específicas. Para facilitar su comprensión tomaré el ejemplo característico de Julián, cuya amplia ruta, con un periodo de migración de 39 años, presenta las diferentes temporalidades y espacialidades que se pretenden caracterizar.

\subsection{El tiempo del trabajo estacional}

Este movimiento se aproxima a lo que en la literatura se le llama ciclo pendular (Carton y Lara-Flores, 2004) y se refiere a aquella movilidad cuya temporalidad se apega a la de una estación de cultivo, o bien, a un conjunto de temporadas de cultivo en los campos del noroeste mexicano o el suroeste estadounidense.

Las temporadas de cultivo, desde la perspectiva del trabajador migrante, se refieren al periodo de trabajo intenso. Así, aunque el ciclo de cultivo del tomate empiece desde marzo con la siembra, es hasta mayo cuando inicia la temporada del corte. En general, los entrevistados hablan de estaciones de trabajo de cuatro o seis meses, las cuales, al alternarse con el empleo en otros cultivos se completa a veces en un año.

Por ejemplo, Carmen, una trabajadora jornalera de San Miguel Tlacotepec, Oaxaca, migró a Sinaloa a los ocho años para cuidar a su hermanita, una bebé de meses, en tanto sus padres trabajaban en el campo. Las temporadas de cosecha duraban seis meses y regresaban recurrentemente a su pueblo de origen. Así estuvieron durante cuatro ańos, hasta que ampliaron el circuito hacia Sonora, para entonces Carmen ya tenía 12 ańos y empezó a trabajar en Sonora y Sinaloa en la siembra de semillas de tomates en invernadero, donde pasaban temporadas de tres o seis meses en cada lugar, de tal manera que la temporada fuera del pueblo se alargó.

A los 14 años se juntó con un joven de 16 años y se independizaron del círculo paterno para tomar el rumbo más al norte, hacia San Vicente Camalú, Baja California, donde llegaron al campamento del Aguaje del Burro y empezaron a trabajar en el cultivo del tomate.

El retorno al lugar de origen o a otro lugar es un evento fundamental que marca el límite de la estación de trabajo. En la biografía de Julián estos movimientos estacionales para trabajar en la agricultura aparecen en dos momentos muy distintos de su vida: cuando es muy joven, y una vez que forma una familia y se establecen en San Quintín.

Por ahora sólo se describe el primero: Julián sale de San Mateo Liebres, en la mixteca oaxaqueña, cuando todavía es un niño. A los 12 años establece por tres años una ruta estacional entre Sinaloa y San Mateo. En ese periodo, su ocupación es la de pizcador de tomate en Sinaloa. Cuando 
retorna a su pueblo su ocupación no es tan clara, ya que no siempre hay un trabajo asalariado. En las diferentes estancias se dedican principalmente a la vida familiar, al arreglo de la vivienda, a la ayuda a sus parientes en el arreglo y trabajo de sus tierras, con empleos no siempre muy definidos.

\subsection{El tiempo de la circulación: familia y trabajo}

Los movimientos estacionales entre lugares específicos mezclan lógicas sociales que incluyen la vida del trabajo y la vida familiar, así como los tiempos asociados a ellos. En la literatura, el concepto de circulación se asocia con el movimiento entre dos o más residencias temporales y el retorno a un punto geográfico inicial.

La distinción entre la migración circular y la migración circular permanente (Carton y Lara-Flores, 2004) presenta algunas dificultades cuando se enfoca en el horizonte biográfico. Los hallazgos de esta investigación señalan que siempre hay un horizonte temporal para la circularidad que responde a una etapa de la vida del individuo; así el fin e inicio de la circulación está marcado por una unidad temporal biográfica. Una temporada de migración pendular puede constituir un circuito. Este es el caso del circuito que por ocho ańos recorrió un migrante de Ocotlán, Oaxaca, viajando cada seis meses a Ruiz Cortinez, Sinaloa.

Otro tipo de circuito sería el que estableció nuestro caso-ejemplo (Julián) entre San Quintín y Del Mar, California, durante dos periodos que suman 17 ańos con una interrupción de dos años en los cuales Julián trabajó en San Quintín. En las entrevistas, los jornaleros refieren periodos de distinta duración en los que viven entre Sinaloa y San Quintín; esto lo hemos encontrado particularmente entre los habitantes de cuarterías y campamentos, muchos de ellos consideran las cuarterías como su hogar.

El concepto de tiempo circular puede incluir el retorno al lugar de origen o un nuevo lugar de residencia que se considera el hogar. Este último punto es un matiz muy importante de incluir en el concepto de retorno en poblaciones con una alta movilidad geográfica. Este periodo incluye los tiempos de trabajo no asalariado, de inactividad o de ocupación en otros empleos en el lugar de origen, los tiempos de espera en los lugares de trabajo, o bien, los tiempos de traslado y espera para cruzar la frontera mexicana, los cuales se prolongaban hasta por meses.

El tema del cuidado de los hijos o de hermanos menores se suscita con mayor frecuencia entre las mujeres: Carmen y Rosa tenían viajes cíclicos entre sus localidades de origen y algún punto de la migración, o desde San Quintín hacia el lugar de origen cuando sus padres las llevaban a los campos, no con la idea de trabajar, sino para cuidar a sus hermanos pequeños o enfermos. 
Ya como mujeres mayores, casadas y con hijos, Carmen y Rosa experimentan una movilidad específicamente para ir a dejar a los hijos con la madre que vivía en el pueblo y ellas poder seguir con su camino migratorio. Carmen dice: "la primera vez que me trajeron al norte venía con mis papás, porque venía a cuidar a mi hermanita, la... que en esos tiempos era la bebé...”. En tanto, Rosa estableció un circuito anual entre San Quintín y su pueblo en Oaxaca para ir a visitar cada año a su hija que había encargado con su madre, debido a que ella no podría hacerse cargo por tener otra pareja y más hijos.

El caso de Carmen me permitirá ilustrar esta temporalidad asociada con la vida familiar y el trabajo, incluyendo los tiempos de espera marcados genéricamente. Carmen y su esposo llegaron a San Quintín en los ańos ochenta, cuando todavía los ciclos de cosecha estaban muy marcados, por lo que, una vez terminada la temporada, regresaban a Sinaloa (al campo Nogalitos) a trabajar en el chile morrón u otras verduras, con retornos cada vez más esporádicos al pueblo de origen.

Finalmente en la década de los noventa, cuando la temporada de cultivo se amplió todo el año, Carmen, su pareja y sus hijos disminuyen la movilidad y se asientan en una colonia de la delegación Vicente Guerrero; el esposo de Carmen inicia la migración hacia California para trabajar en los campos agrícolas. La familia decidió establecerse con unos parientes en Tijuana, donde ella espera nueve o diez meses a que su esposo regrese a visitarla; los periodos de visitas se van alargando y ella decide volver al valle de San Quintín, regresar al trabajo agrícola y esperar los eventuales retornos del esposo.

\subsection{El tiempo de la residencia}

Esta temporalidad se define como el periodo que va desde el primero al último cambio de lugar de residencia, incluye el tiempo biográfico completo del individuo asociado con la movilidad. Este tiempo se puede ejemplificar con la biografía de Julián, quien sale por primera vez a los 12 años de edad de su pueblo natal rumbo al campo Pénjamo, Sinaloa. A los 15 años se va a Caborca donde experimenta la estación de cultivo cuatro meses y regresa a San Mateo, su pueblo natal; durante tres ańos repite este movimiento. A los 18 años, mientras está en Caborca, decide irse a La Paz, Baja California Sur, donde se queda dos años para luego irse al valle de San Quintín a los 20 años, ahí permanece un año y luego viaja a Estados Unidos donde se queda otro año; vuelve a San Quintín donde pasa uno o dos meses y a los 23 ańos va de nuevo a Estados Unidos.

A partir de este momento establece un periodo de 17 ańos, durante los cuales va y viene entre California y San Quintín. En este momento 
de su vida ubica al valle como su lugar de residencia donde vive su esposa y durante ese tiempo nacen sus hijos, por lo que regresa por temporadas de tres o seis meses. En 1996, a los 44 años de edad, regresa a San Quintín donde permanece en forma continua hasta el momento de la entrevista. Actualmente tiene 51 años, de los cuales 29 los ha vivido cambiando de residencia. El regreso a San Quintín fue por asuntos de salud, ya que no veía bien y habían cerrado el campo donde trabajaba para unos empresarios japoneses recolectando tomates en California.

En resumen, la historia migratoria de Julián nos permite observar temporalidades que van de meses a uno o varios años a lo largo de la vida del individuo. Es decir, durante 29 años esta persona tuvo ocho residencias distintas, viviendo en colonias, campamentos, cuarterías, hoyos recubiertos por plástico y, hasta hace aproximadamente cuatro años, construyó una casa en San Quintín y otra en Oaxaca con el dinero que acumuló en Estados Unidos y con el trabajo de su esposa e hijos en San Quintín.

\section{La multiespacialidad o localización de la migración}

Las múltiples temporalidades descritas antes implican, a su vez, la experiencia de varios lugares de residencia o de paso a lo largo de la vida del entrevistado. La importancia de fijar la atención en los lugares es distinguir el tipo de relaciones sociales que determinan la vida de los individuos en cada uno de ellos. La experiencia de los lugares parece distinta dependiendo de la temporalidad de la estancia y del hecho de que el lugar de trabajo no sea el mismo en donde transcurre la vida familiar.

En ciertos periodos de la vida de los entrevistados, los lugares de trabajo son distintos de los de la vida familiar y comunitaria, esta separación o fragmentación tiene importantes consecuencias en la constitución de las experiencias de vida de estos individuos y en las condiciones de vulnerabilidad de su existencia. Lo que las biografías nos mostraron fue que los distintos lugares laborales, de residencia y de paso se articulan gracias a la movilidad poblacional y, en conjunto, permiten el funcionamiento de este tipo de regiones agrícolas, especialmente, las del noroeste como el pacífico estadounidense (California, Oregón y Washington).

Uno de los aspectos más importantes a destacar es que esos lugares condensan espacialidades distintas en la medida que implican universos de relaciones sociales diferenciadas, a veces fragmentadas $y$, otras tantas, integradas. 


\subsection{Lugares de trabajo}

Cuando los entrevistados rememoran su residencia como lugar de trabajo, regularmente se refieren a la vida en campamentos o en cuartos de renta. De tal forma que el lugar era un espacio eminentemente laboral con escasas relaciones familiares y comunitarias, y cuando existía la vida familiar, su dinámica se ceñía a la lógica laboral, estrictamente.

Los jornaleros viven en tal o cual lugar porque trabajan ahí, pero cuando el trabajo se acaba es necesario moverse a otro sitio para ocuparse en otras actividades. Esto se puede ejemplificar con el caso de Julián, quien, como ya lo mencioné, llega a los 12 años al campo Pénjamo, se queda a vivir en la casa de una familia del mismo pueblo y trabaja pizcando tomate durante tres años. A los 15 años sigue rumbo a Caborca para trabajar en la cosecha del algodón y vive rentando un cuarto durante dos años. En esos años, Julián vive entre la cuartería de Caborca y su casa, en el pueblo de San Mateo Liebres. A los 18 ańos, se entera, por unos amigos, que hay trabajo en la pizca de tomate en la Paz, Baja California Sur y decide ir allá, donde se instala por dos años con un paisano en unos cuartos de madera dentro de un campamento.

El recuerdo de estos lugares se asocia con la vida del trabajo agrícola. Las relaciones sociales significativas son de amistad, de paisanaje y laborales informales con patrones o intermediarios como los capataces y mayordomos. Los viajes constantes de un lugar a otro pueden hacerse solo o en grupo; en el relato cobra especial énfasis el regreso de Julián a San Mateo Liebres.

En ocasiones, Julián menciona sólo el regreso a la casa o al pueblo, en tanto que la recolecta o pizca podía implicar lugares distintos. Estos ocho ańos de viajes constantes, desde los 12 a los 20 ańos, constituyen un periodo de intenso trabajo y movilidad en la vida de Julián, donde el pueblo sigue siendo la referencia de hogar.

Aquí, como lo señala Besserer (2007), cada cultivo (la fresa, el tomate, la uva) simboliza tiempos y lugares específicos. Así, cuando los entrevistados decían: "nos fuimos a la pizca del tomate y luego seguimos a la fresa", aluden a tiempos y lugares particulares de su biografía y de la ruta de migración de los flujos humanos de los que ellos forman parte.

Es importante decir que con el asentamiento aunado a los cambios en las temporadas de cultivo, este patrón narrativo se ha alterado ya que en un lugar puede haber tomate y fresa, sólo que en tiempos distintos.

Otra forma de especificar los lugares de trabajo es a través de la conexión de los movimientos, esto a partir de los sitios que mencionan los entrevistados, entre ellos, los ranchos o empresas donde laboraron. Por ejemplo, Victoriano, un trabajador zapoteco, menciona dos viajes a Estados Unidos 
desde San Quintín, y en las dos ocasiones su lugar de llegada es el rancho El Diablo, sin ninguna otra referencia geográfica: "cruzamos por la laguna, decían que era el mar... y llegamos al rancho El Diablo... luego íbamos a dormir al monte... ahí estábamos un tiempo, luego regresábamos de nuevo acá a rancho Seco... [en el valle de San Quintín]...”. Él, como otros trabajadores agrícolas mexicanos, se refugiaban en las laderas de los cerros en el sur de San Diego, durmiendo en casas de cartón improvisadas.

\subsection{Los lugares de la circulación}

En un primer acercamiento, la ruta de la circulación se puede definir por la lógica de funcionamiento de un mercado de trabajo que integra lugares de reproducción y reclutamiento de la fuerza de trabajo y el lugar de producción agrícola. Sin embargo, esta ruta también puede observarse desde la perspectiva del individuo.

A partir de las entrevistas se puede advertir que el conjunto de movimientos, por ejemplo, de un periodo de nueve años, parece definirse por sucesos en la vida del individuo como el casamiento y la formación de una familia; o bien, la muerte de los padres o la estancia de los hijos en la región migratoria.

Por ejemplo, el caso de Julián, en una de las estancias de meses en San Quintín, conoce a la mujer que es actualmente su esposa, quien es del mismo pueblo, a partir de ese momento la temporalidad de sus desplazamientos cambia notoriamente así como el radio de sus desplazamientos. Después de casarse, va de nuevo a Estados Unidos, dejando a su esposa en el valle de San Quintín, quien también trabajaba en el campo, establece durante 17 años un patrón de movilidad entre San Quintín y California hasta que decide regresar a trabajar al valle por problemas de salud y escasez de trabajo en California.

Si bien la movilidad hacia California tenía una lógica de trabajo o empleo, sus retornos constantes a San Quintín obedecían al deseo de estar con su familia y de resolver las necesidades familiares como arreglar el terreno, la vivienda y estar con los hijos. Durante este tiempo compraron un terreno y construyeron un hogar en San Quintín, también edificaron una casa para sus padres en Oaxaca. Durante esos 17 años, Julián considera que su hogar estaba en San Quintín y su trabajo en California.

El caso de Rosa nos ofrece otro circuito que involucra a San Quintín, como residencia y trabajo, y la localidad San José de las Flores, en el municipio Putla Villa de Guerrero, Oaxaca, de donde es originaria y donde se quedó su madre. Este circuito lo estableció desde hace diez años, Rosa vio una posibilidad de disminuir los gastos en su hogar, enviando a 
su hija Antonia de seis años con su abuela materna, quien le ofrecía la posibilidad de que la nińa estudiara. Desde entonces Rosa envía dinero a su madre para la manutención de Antonia y va cada año a visitarla.

\subsection{Los lugares de la migración: de residencia, de paso, espera y cruce de la frontera estatal}

$\mathrm{Al}$ analizar las historias de migración obtenidas a partir de las entrevistas; además de los distintos lugares de residencia como trabajadores, como nuevos colonos o como miembros de una familia o comunidad, se encontró otro tipo de lugares por los que transitan los jornaleros y que marcan su experiencia espacial biográfica. Estos lugares son aquellos que sólo son mencionados como de paso en un trayecto específico, como es el caso de Julián, quien refiere una estancia de un mes en San Quintín en su historia migratoria.

En el contexto de la trayectoria global, en ese momento San Quintín es considerado sólo como lugar de paso donde se contactaron a personas para continuar el viaje a Tijuana para cruzar la frontera. En estos espacios sobresalen los sitios de cruce de la frontera mexicano-estadounidense, los cuales cobran especial relevancia en la narración de los entrevistados, en la medida que generan relaciones sociales nuevas como son las que se establecen con los polleros, con la migra o los patrones del otro lado. La experiencia que parece marcar estos lugares es el riesgo y el peligro.

En el caso de Julián, por ejemplo, en uno de los cruces que realizó durante los 17 años que pasaba constantemente hacia California por Tijuana, y cuyas estancias en esta ciudad podían durar hasta dos meses, fue asaltado y herido por un grupo de jóvenes baja pollos. ${ }^{19}$ A consecuencia de ese ataque perdió parte de la vista, empezó con un problema constante para trabajar y para desplazarse por su propia cuenta. La distinción de los lugares de cruce señala una experiencia espacial diferenciada entre la nacional y la internacional.

El caso de Carmen puede servir para contrastar la idea de cruce y espera, ya que ella se establece en Tijuana mientras su esposo va y viene a California. Para ella, esta ciudad fronteriza fue un lugar de espera para el retorno mensual de su esposo; hasta que los regresos se van haciendo cada vez más esporádicos y ella decide regresar con su familia a San Quintín y trabajar como jornalera en el campo.

${ }^{19}$ Así se nombra coloquialmente a los asaltantes de migrantes en las zonas de cruce. 


\section{Los movimientos temporales, las estancias continuas y el proceso de asentamiento}

Al hacer el análisis de las historias de migración construidas libremente por el entrevistado alrededor de los ejes mencionados, lo que se detectó fueron temporalidades distintas en su propio recuento de migración. Los movimientos fueron relatados como episodios de vida que respondían a lógicas distintas, por ejemplo, en el tiempo de trabajo estacional, el episodio de vida estaba marcado por la lógica de la producción agrícola de exportación, en tanto que el tiempo circular combinaba en un episodio de vida, lógicas de urgencia familiar o individual, como regresar con la familia (la muerte de algún pariente, el nacimiento de un hijo, una festividad, etc.) o a resolver un problema vecinal o comunitario.

Este tiempo intermedio -llamémosle los periodos de circulaciónregularmente era mayor de un año. Esta referencia temporal intermedia correspondía a una temporada más amplia de la vida que combinaba varios cambios de residencia, con los tiempos de trabajo y los ciclos de la vida familiar. Es importante considerar la reconstrucción narrativa de la movilidad geográfica por lo que esos movimientos estacionales y circulares tienen como referencia constante el total de experiencia migratoria que constantemente ubicaba al entrevistado en el presente con la mirada al pasado.

Tanto para los periodos de trabajo estacional menores de un año como para los periodos de circulación, mayores de un año, la idea del retorno desempeńa un papel central como evento marcador entre un episodio de vida y otro. La idea de retorno ubicaba el hogar familiar, ya fuera el de orientación o procreación, dependiendo del momento biográfico del entrevistado y el cual no siempre era en el lugar de origen. En las historias de vida había un quedarse y un irse y, conforme el tiempo de alojamiento en un lugar era más amplio y se suscitaba la llegada de familiares, este nuevo lugar se convertía en la referencia para la movilidad.

Las entrevistas se realizaron a personas que se consideran residentes en alguna colonia de las dos delegaciones más pobladas del valle de San Quintín. En general, contaban con un terreno en proceso de regularización y pocas de ellas estaban rentando, por lo que la residencia en la nueva colonia se convierte en la referencia de hogar para su movilidad actual, apareciendo la idea de permanencia continua a partir de ciertos eventos como la llegada de familiares, la posesión de un terreno, el pago de servicios, la asistencia de los hijos a la escuela, la puesta en marcha de un negocio, sucesos que se convierten en indicadores de permanencia.

En síntesis, son comunes los distintos tipos de movilidades en la historia de un individuo regularmente mayor de 18 ańos, que combina los 
tiempos cortos de la producción agrícola con los tiempos intermedios de la circulación, influidos por la vida familiar -en sus distintas etapas- y con los tiempos de otras ocupaciones en los lugares de origen, o en los nuevos lugares de residencia durante las temporadas bajas de la producción agrícola, como es el caso de la construcción, el comercio o la colecta de piedras en la playa.

El asentamiento en un lugar de migración, indicado por una permanencia mayor, no es suficiente si no se acompaña de indicadores de orden social como la posesión de un terreno, el pago de servicios, la asistencia de los hijos a la escuela, la participación comunitaria y las condiciones de salud y edad del migrante. Hablar de asentamiento es hablar de apego al nuevo lugar. Lo que la literatura reporta constantemente en encuestas transversales como migrantes indígenas en la agricultura, con edades que proporcionalmente se concentran entre los 16 y 29 años de edad, corresponde a un momento de intensificación de la movilidad y del trabajo en la trayectoria vital de los individuos.

\section{Conclusiones}

El tema de la especialización laboral ha sido una constante en la literatura sobre migración indígena, para el caso del valle de San Quintín, está asociada con el trabajo en los cultivos como jornalero. Tanto en colonias como en campamentos, la población que habla lengua indígena se dedica a la agricultura en una proporción mayor que los que no pertenecen a algún grupo étnico.

En el análisis de los itinerarios de vida no se logró encontrar tal especialización por cultivo. En general, en la trayectoria encontramos que hay una variedad de cultivos de frutas y verduras frescas, y que ello, más bien, corresponde a las distintas estaciones de cultivo en el año, en cada una de las regiones o lugares donde residían los migrantes (Ebimbre, 2005).

Hay un incremento en la flexibilidad de estos trabajadores para entrar y salir de los campos de cultivo y combinarlos con actividades remuneradas en otros sectores como el comercio o la extracción, particularmente la recolección de piedras de mar para exportarlas a California. Esta especialización y flexibilización laboral de la población indígena sigue una lógica no sólo del mercado laboral, sino también de su vida familiar. La salida y entrada del mercado de trabajo agrícola depende de las edades y número de integrantes del hogar; esta organización se realiza prácticamente por semana, con periodos intensos durante la temporada alta de cosecha para ciertos cultivos como el tomate y la fresa.

Pero también tiene una lógica de agotamiento físico de los trabajadores, pues el trabajo intenso de siete días a la semana exige dos días, ya 
sea de descanso o por enfermedad. Es posible decir todavía que la especialización laboral de los migrantes indígenas funciona en torno al sector agrícola y la posición de peón en el trabajo se ha roto en torno al tipo de cultivo y de contratación (tarea, jornada o pieza), número de patrones e incorporación al empleo, ya que el enganche es cada vez menos frecuente como resultado del asentamiento.

Hay una creciente flexibilización que opera en forma muy amplia a lo largo de la biografía de los individuos, ya que cada vez hay una mayor combinación de empleos en sectores distintos, o bien, en el mismo sector en otros lugares fuera de la región, como es en California y Oregón. Esta flexibilización va al terreno del grupo, a través de la capacidad de reemplazo generacional, como parte de las estrategias de reproducción de los hogares en la medida que existen nuevos integrantes que pueden entrar a sustituir o relevar a otros miembros, no sólo en tiempos de enfermedades o de movilidad geográfica, sino en el ritmo de intensidad que las nuevas formas de producción global exigen a los trabajadores y sus hogares.

El estudio biográfico no sólo permite dar cuenta de las distintas lógicas sociales que dominan el comportamiento de los individuos, sino también descubrir cómo están conectadas con las estructuras sociales que definen las opciones individuales. De tal forma que, al observar las lógicas que organizan la movilidad y migración de estos migrantes, es posible conocer las negociaciones -entre las lógicas de los mercados de trabajo con necesidades de mano de obra en tiempo y forma- con las lógicas familiares o comunitarias.

Los encuentros familiares o la asistencia a las fiestas comunitarias cobran una relevancia similar a la de los traslados para acudir a las cosechas de tomate, o bien, cómo esas distintas movilidades nacionales o transfronterizas tienen sentido en el horizonte residencial que incluye la compra de un terreno o la construcción de una casa en un nuevo lugar. Los individuos organizan el asentamiento en configuraciones familiares tan versátiles como las utilizadas para la movilidad (Carton et al., 2004), desarrollando de manera paulatina apego y arraigo.

A partir de los casos analizados y del caso típico utilizado se pudo conocer la conexión del asentamiento en San Quintín con la migración internacional, en particular a los campos de cultivo de California. Las entrevistas fueron realizadas entre 2000 y 2006, de tal forma que la frontera ya registraba el efecto de las políticas de control para limitar el cruce de México a Estados Unidos. Ya se había establecido la Operación Guardián (1994) y apenas se notaba el efecto del vínculo de la política migratoria con la seguridad nacional estadounidense, a raíz de la caída de las torres gemelas de Nueva York, en septiembre de 2001. 
Estos cambios se expresaban en estancias más largas en Estados Unidos, o bien, en una disminución del cruce de otros miembros de la familia ante el incremento del costo y riesgo para pasar la frontera. El asentamiento en esta zona permite un mayor control de los costos de la movilidad transfronteriza. El análisis en conjunto de estas formas diferentes de construir el territorio: movilidad, espera y asentamiento surge como una necesidad para comprender las múltiples lógicas (Cresswell, 2012) que dominan los itinerarios de estas poblaciones móviles, pero también en proceso de arraigo con horizontes étnicos específicos.

\section{Bibliografía}

Acebo, Enrique del (1996), Sociología del arraigo. Una lectura critica de la teoría de la ciudad, Editorial Claridad, Buenos Aires.

Arroyo-Sepúlveda, Ramiro (2003), "Migración y apropiación productiva entre los jornaleros agrícolas indígenas”, México Indigena, 2 (6), INAH, México, pp. 64-70.

Barrón, María Antonieta (1994), "Migración y empleo en los cultivos de hortalizas de exportación en México" en Alejandro Dabat (coord.) México y la globalización, CRIM-UnAm, México, pp. 261-287.

Besserer, Federico (2007), "Luchas transculturales y conocimiento práctico" en Mariana Ariza y Alejandro Portes (coords.), El país transnacional. Migración mexicana y cambio social a través de la frontera, unam, México, pp. 323-347.

Carton de Grammont, Hubert y Sara María Lara-Flores (2004), Encuesta a hogares de jornaleros migrantes en regiones hortícolas de México: Sinaloa, Sonora, Baja California Sur y Jalisco, Cuadernos de investigación núm. 30, unAM, México.

Carton de Grammont, Hubert, Francis Marie Hurbert, Sara Lara-Flores y Martha Judith Sánchez (2004), "Migración rural temporal y nuevas configuraciones familiares: (los casos de Sinaloa, México; Napa y Sonoma, eeuu)", en Marina Ariza y Orlandina de Oliveira, Imágenes de la familia en el cambio de siglo, universo familiar y procesos demográficos contemporáneos, UnAM, México, pp. 357-368.

Coubès, Marie Laure (2005), "La collecte des systèmes résidentiels dans une enquête biographique: un exemple de questionnaire auprès de travailleurs agricoles de San Quintín (Basse Californie, Mexi- 
que)", ponencia presentada en el XXV Congreso Internacional de Población, 18-23 de Julio, Tours.

Coubès, Marie Laure, Laura Velasco y Christian Zlolniski (2013), "Residential settlement and mobility in the san quintin valley: methodological reflections on an interdisciplinary study", en Liliana Rivera-Sánchez and Fernando Lozano-Ascencio, The practice of research on migration and mobilities, vol. 14, Springer, London, pp. 19-42.

Conepo-Colef (Consejo Estatal de Población y Colegio de la Frontera Norte) (2002), "Encuesta sociodemográfica y de migración del valle de San Quintín”, Conepo-Colef, México.

Conepo-Colef (Consejo Estatal de Población y Colegio de la Frontera Norte (2003), "Encuesta a jornaleros agrícolas en campamentos del valle de San Quintín”, Conepo-Colef, México.

Cresswell, Tim (2012), "Mobilities II: Still”, Progress in human geography, 36 (5), Royal Holloway-University of London, London, pp. 645653.

Cresswell, Tim (2013), "A theory of migration", Transfers, 3 (1), Berghahn Journals, New York, pp. 6-7.

Cresswell, Tim (2014), "Place" in Paul Cloke, Philip Crang and Mark Goodwin (eds.), Introducing human geographies, Routledge-Taylor \& Francis Group, Nueva York-London, pp. 249-261.

Ebimre (Encuesta Biográfica de Movilidad Residencial y Empleo en San Quintín) (2005), El Colegio de la Frontera Norte, Mexicali.

Fox, Jonathan y Gaspar Rivera (eds.) (2004), Indigenous mexican migrants in the United States, Center for Comparative Immigration-University of California San Diego, La Jolla.

Heyman, Joshiah (2012), "Construcción y uso de tipologías: movilidad geográfica desigual en la frontera México-Estados Unidos”, en Marina Ariza y Laura Velasco, Métodos cualitativos y su aplicación empirica: por los caminos de la investigación en la migración internacional, Colef-unam, México, pp. 419-454. 
INEGi (Instituto Nacional de Estadística y Geografía) (1990), Censo General de Población y Vivienda Baja California, Dirección General de Estadística, Inegi, México.

INEgi (Instituto Nacional de Estadística y Geografía) (2000), Censo General de Población y Vivienda Baja California, Dirección General de Estadística, Inegi, México.

Lara-Flores, Sara María (2003), "La migración jornalera, antesala de las migraciones ilegales hacia Estados Unidos: el caso de Coatecas Altas un pueblo indígena de Oaxaca”, México Indígena, 2 (6), INAH, México, pp. 6-11.

Lara-Flores, Sara y Hubert Carton de Grammont (2003), “Jornaleros agrícolas y migración temporal en las empresas hortícolas mexicanas", Este País, núm. 148, unam, México, pp. 63-68.

Lara-Flores, Sara María (2008), "Espacio y territorialidad en las migraciones rurales. Un ejemplo en el caso de México", en Pablo Castro-Domingo, Dilemas de la migración en la sociedad posindustrial, UAem-uam-Conacyt-Miguel Ángel Porrúa, México, pp. 17-38.

Martínez-Veiga, Ubaldo (2001), El ejido. Discriminación, exclusión social y racismo. Los libros de la Catarata, Madrid.

Rubio, Miguel Ángel, Saúl Millán y Javier Gutiérrez (coords.) (2000), La migración indígena en México. Estado del desarrollo de los pueblos indígenas en México, Instituto Nacional Indigenista-Programa de las Naciones Unidas para el Desarrollo, México.

Sagarpa (Secretaría de Agricultura, Ganadería, Desarrollo Rural, Pesca y Alimentación) (1992-2003), Base de datos sobre producción agrícola en el valle de San Quintín, Avance, 1980-2003. Programación 1992-2003. Procesamientos del Proyecto "Migración, trabajo agrícola y relaciones étnicas: la articulación entre lo global, nacional y local en el valle de San Quintín", Conacyt-El Colegio de la Frontera Norte, México.

Scudder, Thayer (1985), "A sociological framework for the analysis of newland settlements", en M. Cernea (ed.) Putting people first. Sociological variables in rural development, Oxford University Press, Oxford, pp. 121-153. 
Velasco, Laura (2002), El regreso de la comunidad: migración indígena y agentes étnicos. Los mixtecos en la frontera México-Estados Unidos, Colmex-Colef, México.

Velasco, Laura (2005), Mixtec transnational identity, University of Arizona Press, Tucson.

Velasco, Laura (coord.) (2010), Tijuana indigena. Estudio sobre las condiciones de vida e integración social de la población indígena a la ciudad, Cuadernos de investigación, Comisión Nacional para el Desarrollo de los Pueblos Indígenas, México.

Zabin, Carol (1993), "Labor market interdependence between México and the Us: wage convergence or new gender and ethnic hierarchies in California and Baja California agriculture?", Center for Latin American Studies, Tulane University, New Orleans, Manuscrito.

Recibido: 7 de abril de 2010. Reenviado: 5 de octubre de 2011. Aceptado: 19 de octubre de 2013.

Matilde Laura Velasco-Ortiz. Mexicana. Doctora en ciencias sociales con especialidad en sociología por El Colegio de México. Es investigadora del Departamento de Estudios Culturales de El Colegio de la Frontera Norte, donde labora desde 1991. Además se ha desempeñado en dicha institución como docente desde 1999 hasta la fecha, donde ha impartido cursos a nivel de grado y posgrado sobre: identidad y migración, metodología de investigación, la cuestión étnica en los estados nacionales modernos, identidad, etnicidad y multiculturalidad, entre otros. Cuenta con una extensa producción de capítulos de libros y artículos de revistas con arbitraje nacional e internacional. Entre sus principales publicaciones destacan, coordinado con Marina Ariza: Métodos cualitativos y su aplicación empirica. Por los caminos de la investigación sobre migración internacional, El Colef-unam, México, 546 pp. (2012), en coautoría: Mexican voices of the border region, Temple University Press, Philadelphia, 216 pp. (2011); Migración, fronteras e identidades étnicas transnacionales (coord.), El ColefMiguel Ángel Porrúa, México, 344 pp. (2008); Mixtec transnational identity, University of Arizona Press, Tucson, 272 pp. (2005); Desde que tengo memoria: narrativas de identidad en comunidades de indigenas migrantes, El Colef-Fonca, México, 303 pp. (2005); entre otras. 